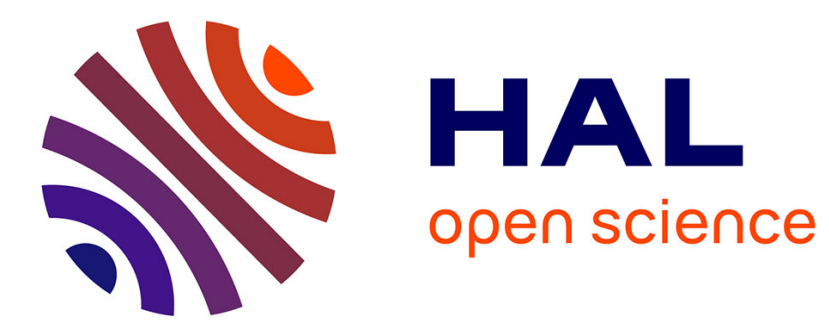

\title{
ESPRIT Method Enhancement for Real-time Wind Turbine Fault Recognition
}

\author{
Saad Chakkor, Mostafa Baghouri, Abderrahmane Hajraoui
}

\section{To cite this version:}

Saad Chakkor, Mostafa Baghouri, Abderrahmane Hajraoui. ESPRIT Method Enhancement for Realtime Wind Turbine Fault Recognition. International Journal of Power Electronics and Drive System (IJPEDS) , 2015, pp.552-567. hal-01152449

\author{
HAL Id: hal-01152449 \\ https://hal.inria.fr/hal-01152449
}

Submitted on 18 May 2015

HAL is a multi-disciplinary open access archive for the deposit and dissemination of scientific research documents, whether they are published or not. The documents may come from teaching and research institutions in France or abroad, or from public or private research centers.
L'archive ouverte pluridisciplinaire HAL, est destinée au dépôt et à la diffusion de documents scientifiques de niveau recherche, publiés ou non, émanant des établissements d'enseignement et de recherche français ou étrangers, des laboratoires publics ou privés.

\section{다)(1) $(5$}

Distributed under a Creative Commons Attribution - NonCommercial| 4.0 International 


\title{
ESPRIT Method Enhancement for Real-time Wind Turbine Fault Recognition
}

\author{
Saad Chakkor, Mostafa Baghouri, Abderrahmane Hajraoui
}

University of Abdelmalek Essaâdi, Faculty of Sciences, Department of Physics,

Communication and Detection Systems Laboratory, Tetouan, Morocco

\section{Article Info \\ Article history: \\ Received Nov 12, 2014 \\ Revised Feb 3, 2015 \\ Accepted Feb 20, 2015 \\ Keyword: \\ Diagnosis \\ ESPRIT \\ Real Time \\ Spectral Estimation \\ Wind Turbine Faults}

\begin{abstract}
Early fault diagnosis plays a very important role in the modern energy production systems. The wind turbine machine requires a regular maintenance to guarantee an acceptable lifetime and to minimize production loss. In order to implement a fast, proactive condition monitoring, ESPRITTLS method seems the correct choice due to its robustness in improving the frequency and amplitude detection. Nevertheless, it has a very complex computation to implement in real time. To avoid this problem, a FastESPRIT algorithm that combined the IIR band-pass filtering technique, the decimation technique and the original ESPRIT-TLS method were employed to enhance extracting accurately frequencies and their magnitudes from the wind stator current. The proposed algorithm has been evaluated by computer simulations with many fault scenarios. Study results demonstrate the performance of Fast-ESPRIT allowing fast and high resolution harmonics identification with minimum computation time and less memory cost.
\end{abstract}

Copyright () 2015 Institute of Advanced Engineering and Science. All rights reserved.

\section{Corresponding Author:}

Saad Chakkor,

University of Abdelmalek Essaâdi,

Faculty of Sciences, Department of Physics,

Communication and Detection Systems Laboratory,

Tetouan, Morocco

Email: saadchakkor@gmail.com

\section{INTRODUCTION}

Wind energy has become one of the popular renewable powers all over the world in electricity generation capacity. Wind turbines contain a complex electromecanical system which is prone to defects. Consequently, there is an increase need to implement a predictive monitoring scheme of wind turbines, allowing an early detection of electromechanical faults, in order to avoid catastrophic damage, to reduce maintenance costs, to ensure continuity of production and to minimize downtime. It means that stopping a wind installation for unexpected failures could lead to expensive repair. These faults cause a modulation impact in the magnetic field of the wind generator, which is characterized by the appearance of a significant harmonics (peaks) in the stator current spectrum. For this reason, most of the recent researches have been oriented their interest toward electrical monitoring, with focus on frequency analysis of stator current (CSA). This technique is more practical and less costly [1]-[4]. Furthermore, with recent digital signal processor (DSP) technology developments, motor and generator fault diagnosis can now be done in real-time [1]. ESPRIT is one high resolution or subspace method (HRM) which is widely adopted in electromechanical machine diagnosis. It can be used for spectral estimation [3], [5]-[6]. This algorithm allows very high spectral detection accuracy and a high resistance to noise compared to others methods like MUSIC and Root-MUSIC. Contrariwise, it require long computation time to find more frequency estimates when the autocorrelation matrix is large and the order of sampled data dimension increase. This fact makes its application in real time detection very limited despite its high precision. This article presents an ameliorated version of ESPRIT-TLS 
method for fast wind turbine faults detection and diagnosis based on a band pass filtering technique. The proposed improvement allows many advantages: reduction of computation time, saving of memory space and accuracy increase in a specified frequency bandwidth. The paper is organized as follows: the problem is formulated in section 2, the stator current signal is presented in section 3, and then section 4 describes wind turbine fault models. While section5 focuses on ESPRIT method theory, section6 explains in details the proposed approach to enhance original ESPRIT algorithm. Simulation results are presented and discussed in Section 7. Finally, conclusions with future work are drawn in the last section.

\section{RELATED WORK}

Many research studies applying enhanced and advanced signal processing techniques have been used in the motor and generator stator current to monitor and to diagnose prospective electromechanical faults. The classical methods like periodogram and its extensions which are evaluated through a Fast Fourier Transform (FFT) are not a consistent estimator of the PSD because its variance does not tend to zero as the data length tends to infinity. Despite this drawback, the periodogram has been used extensively for failure detection in research works [4], [6]. The (FFT) does not give any information on the time at which a frequency component occurs. Therefore, the Short Time Fourier Transform approach (STFT) is used to remove this shortcoming. A disadvantage of this approach is the increased sampling time for a good frequency resolution [7]. The discrimination of the frequency components contained within the signal, is limited by the length of the window relative to the duration of the signal [8]. To overcome this problem, in [9] and in [10] Discrete Wavelet Transform (DWT) is used to diagnose failures under transient conditions for wind energy conversion systems by analyzing frequencies with different resolutions. This method facilitates signal interpretation because it operates with all information contained in the signal by time-frequency redistribution. One limitation of this technique that it gives a good time resolution and poor frequency resolution at high frequencies, and it provides a good frequency resolution and poor time resolution at low frequencies [4], [11]. Recently, high resolution methods (HRM) are applied to detect more frequencies with low SNR. In fact, MUSIC and ESPRIT techniques with its zooming extensions are conjugated to improve the identification of a large number of frequencies in a given range [12], [13]. In [14] a comparative performance analysis of (HRM) is made. This study has demonstrated that ESPRIT method has a high accuracy which exceeds all other algorithms even with the existence of an annoying noise. Moreover, these algorithms are based on an eigenanalysis of the autocorrelation matrix of a signal corrupted by noise. This decomposition requires a long computation time mainly when the size of the autocorrelation matrix and the number of data samples increase. In [15] a rank reduced ESPRIT technique is proposed to transform it into simplified lowcomplexity algorithm. However, this method presents performance deterioration mainly with the SNR decreasing and lowers harmonic amplitudes. Moreover, it has not focused on the minimization of the computational time execution for real applications. This work proposes a solution to overcome the complexity cost of ESPRIT-TLS in the purpose of its use in a real time wind turbine monitoring.

\section{STATOR CURRENT MODEL}

The possibility of detecting faults in electrical induction machines has been extensively studied using vibration surveillance. However, the reliability of the results of this technique is strongly depending on the accelerometers position placed in these machines according to the vertical, axial and radial axes. This is in fact the main drawback of this technique of vibration. In addition, it may also be affected by the speed of the machine, especially when the mechanical components are damaged. Thereafter, these procedures are expensive because they require additional transducers. Their use makes sense only in the case of large machines or highly critical applications. Nevertheless, monitoring of stator current lines appears to be the most interesting and most attractive mode to support fault recongnition because the stator current is very accessible and it can be measured directly. In addition, it can be used to diagnose mechanical and electrical defects. This offers a cost effective and acceptable alternative based on the CSA technique. In fact, to build a correct detection of the wind turbine fault modulations and signatures in the stator current, it is necessary to construct a complex signal associated with the real one. This analytical signal model describes precisely the behavior and the evolution of the real stator current. It contains relevant fault information. For these reasons it is often used for command purposes. The studied wind generator stator current will be denoted by the discrete signal $i[n]$. This signal is considered as a sum of $L$ complex sinusoids and white noise. It is obtained by sampling the continuous time current every $T_{s}=1 / F_{s}$ seconds. The induction generator stator current $i[n]$ in presence of mechanical and/or electrical faults has a data model which can be expressed as follows [10]: 


$$
i[n]=\sum_{k=1}^{L} I_{k} e^{j\left(2 \pi \frac{f_{k}}{F_{s}}, n+\varphi_{k}\right)}+b[n]
$$

Where $i[n]$ corresponds to the $\mathrm{nth}$ stator current sample with $\mathrm{n}=0,1,2 \ldots N_{s}-1 . I_{k}, f_{k}$, and $\varphi_{k}$ are the amplitude, the frequency and the phase of the $\mathrm{k}^{\text {th }}$ complex sinusoid (harmonic components) respectively, $b[n]$ is a gaussian white noise. $F_{s}$ is the sampling frequency and $N_{s}$ is the number of data samples. $L$ represents the number of researched harmonics.

\section{WIND TURBINE FAULT MODELS}

The wind machine is susceptible to diverse electro-mechanical anomalies that involve mostly five components: the stator, the rotor, the bearings, gearbox and/or the air gap (eccentricity) [16]. These defects require a predictive recognition to avert any side effect provoking a breakdown or a fatal spoilage. Because it contains the totally relevant fault information, the stator current spectrum is examined to withdraw the sideband frequency components inserted by the fault. These fault frequencies are located around the fundamental line frequency and are called lower sideband and upper sideband components. This detection technique is used in collaboration with one bit vibration sensors for an early identifying of prospective electromechanical failures which can occurs in any time. A synopsis of wind turbine faults and their related frequencies formulas are presented in Table 1.

Table 1. Wind Turbines Faults Signatures

\begin{tabular}{ccc}
\hline Failure & Harmonic Frequencies & Parameters \\
\hline $\begin{array}{c}\text { Broken rotor } \\
\text { bars } \\
\text { (brb) }\end{array}$ & $f_{b r b}=f_{0}\left[k\left(\frac{1-s}{P}\right) \pm s\right]$ & $k=1,3,5, \ldots$ \\
$\begin{array}{c}\text { Bearing } \\
\text { damage } \\
\text { (bng) }\end{array}$ & $f_{\text {bng }}=\left|f_{0} \pm k f_{i, o}\right|$ & $k=1,3,5, \ldots$ \\
$\begin{array}{c}\text { Misalignment } \\
\text { (mis) }\end{array}$ & $f_{i, o}=\left\{\begin{array}{l}0.4 n_{b} f_{r} \\
0.6 n_{b} f_{r}\end{array}\right.$ \\
$\begin{array}{c}\text { Air gap } \\
\text { eccentricity } \\
\text { (ecc) }\end{array}$ & $f_{\text {ecc }}=f_{0}\left[1 \pm m\left(\frac{1-s}{P}\right)\right]$ & $k=1,3,5, \ldots$
\end{tabular}

Where $f_{0}$ is the electrical supply frequency, $s$ is the per-unit slip, $P$ is the number of poles, $f_{\mathrm{r}}$ is the rotor frequency, $n_{b}$ is the bearing balls number, $f_{\mathrm{i}, \mathrm{o}}$ is the inner and the outer frequencies depending on the bearing characteristics, and $m, k \in \mathbb{N}$ are the harmonic frequency index [4], [9]-[10]. Slip $s$ is defined as:

$$
\begin{aligned}
& s=\frac{\omega_{s}-\omega_{r}}{\omega_{s}} \\
& \omega_{s}=\frac{120 f_{0}}{P} \\
& \omega_{s} \text { is the generator synchronous speed, } \\
& \omega_{r} \text { is the relative mechanical speed of the generator, }
\end{aligned}
$$

These harmonics are extensively used as diagnostic measures in the CSA approach.

\section{ESPRIT METHOD THEORY}

High resolution methods are recently used for fault diagnosis. They can detect and identify the faulty element based on its frequency. The most accurate and efficient technique is ESPRIT which belongs to the subspace parametric spectrum estimation methods. It is based on eigenvector decomposition which aims to separate the observation space in a signal subspace, containing only useful information, and its orthogonal complement, called noise subspace. The rotational invariance between both subspaces allows extracting of the parameters of spectral components present within the investigated waveform [17], [18], [20]. 


\subsection{Autocorrelation Matrix Estimation}

Based on the stator current model defined by the Equation (1), the autocorrelation matrix can be then estimated as follow [19]:

$$
R_{i}=E\left[i(n) \cdot i^{H}(n)\right]=R_{s}+R_{b}=S . P . S^{H}+\sigma_{b}^{2} . I
$$

It is composed by the sum of signal and noise autocorrelation matrices. Where $H$ is the Hermitian transpose, $\sigma_{b}{ }^{2}$ is the variance of the white noise, $I$ is the identity matrix of size $\left(N_{s} \times N_{s}\right)$ and $P$ is the power matrix of the harmonics:

$$
P=\operatorname{diag}\left[I_{1}^{2} I_{2}^{2} \cdots I_{L}^{2}\right]
$$

$S$ is the Vandermonde matrix defined by:

$$
\begin{aligned}
& S=\left[s_{1} \cdots s_{i} \cdots s_{L}\right] \\
& S_{k}=\left[1 e^{j\left(2 \pi \cdot \frac{f_{k}}{F_{s}}\right)} e^{j\left(4 \pi \cdot \frac{f_{k}}{F_{s}}\right)} \cdots e^{j\left(2 \pi \cdot\left(s_{s}-1\right) \cdot \frac{f_{k}}{F_{s}}\right)}\right]^{T}
\end{aligned}
$$

The finite data length of the signal makes the computation of the autocorrelation matrix $R_{i}$ inaccurate. For real purpose, this matrix is unknown and it must be singular. For effective detection, it is necessary to reduce the statistical fluctuations present in estimating the autocorrelation matrix by the averaging [7], [19]. In addition, the accuracy of ESPRIT depends on the dimension $\left(M \leq N_{s}\right)$ of $R_{i}$. It is possible to estimate it from the acquired data samples by the following relationship [7], [19]:

$$
\hat{R}_{i}=\frac{1}{N_{s}-M+1} D \cdot D^{H}
$$

Where $M$ is the data matrix order and $D$ is a Hankel data matrix defined by:

$$
D=\left[\begin{array}{ccc}
i(0) & \cdots & i\left(N_{s}-M\right) \\
\vdots & \cdots & \vdots \\
i(M-1) & \cdots & i\left(N_{s}-1\right)
\end{array}\right]
$$

The dimension of $R_{i}$ should be high enough to have more eigenvalues for noise space and should be low enough to minimize the computation time cost. When the value of $M$ decreases below $N_{s} / 3$, it can be seen the increase of the frequency detection error. Contrariwise, if $M$ increases beyond $N_{s} / 2$, calculation time increases. So, there is a trade-off for the right choice of $M$. Empirically, the value of $M$ is chosen to be bounded as shown in (10) to give a good performance:

$$
\frac{N_{s}}{3}<M<\frac{N_{s}}{2}
$$

In this paper, the autocorrelation matrix dimension $M$ is taken rounded down as follows:

$$
\hat{M}=\text { Round }\left(\frac{N_{s}-1}{2}\right)
$$

Evidently, the number of frequencies $L$ is not a priori known. The frequency signal dimension order (FSDO) $L$ must to be estimated by the minimization of a cost function $M D L(k)$ named minimum description length. In order to obtain a robust estimate, (MDL) criterion is used as shown in the following formula [18] for $k=1,2, \ldots, L$ : 


$$
M D L(k)=-\log \left(\frac{\prod_{i=k+1}^{L} \lambda_{i}^{\frac{1}{L-k}}}{\frac{1}{L-k} \sum_{i=k+1}^{L} \lambda_{i}}\right)^{\rho(L-k)}+\frac{1}{2} k(2 L-k) \log (\rho)
$$

$$
\rho=N_{s}-L-2
$$

Where $\lambda_{i}$ are eigenvalues autocorrelation matrix $R_{i}$. Analytically, the estimate of $L$ can then be expressed in the form:

$$
\hat{L}=\arg _{k} \min (M D L(k))
$$

However, ESPRIT performances are completely degraded by choosing a wrong FSDO value.

\subsection{Eigendecomposition of autocorrelation matrix}

The eigendecomposition of the autocorrelation matrix $R_{i}$ is given by exploiting the eigenvalues $\left\{\lambda_{1}, \lambda_{2}, \ldots, \lambda_{M}\right\}$ and their corresponding signal eigenvectors $\left\{v_{1}, v_{2}, \ldots, v_{M}\right\}[17]$ :

$$
R_{i}=\sum_{k=1}^{N_{s}} \lambda_{k} v_{k} v_{k}^{H}=\underbrace{U_{s} \cdot E_{s} \cdot U_{s}^{H}}_{R_{s}}+\underbrace{U_{b} \cdot E_{b} \cdot U_{b}^{H}}_{R_{b}}
$$

Where:

$$
\begin{aligned}
& U_{s}=\left[v_{1} \cdots v_{L}\right], E_{s}=\operatorname{diag}\left[\lambda_{1} \cdots \lambda_{L}\right] \\
& U_{b}=\left[v_{L+1} \cdots v_{N_{s}}\right], E_{b}=\sigma_{b}^{2} I_{N_{s}-L}
\end{aligned}
$$

$U_{s}$ represents the eigenvectors matrix of the signal space related to the $L$ largest eigenvalues arranged in descending order. Whereas, $U_{b}$ represents the eigenvectors matrix of the noise space related to the $N_{s}-L$ eigenvectors that, ideally, have eigenvalues equal to the variance noise $\sigma_{b}^{2}$. Diagonal matrices $E_{S}$ and $E_{b}$ contain eigenvalues $\lambda_{i}$ corresponding to eigenvectors $v_{i}$.

\subsection{Frequency Estimation}

ESPRIT method is based on the study of the signal subspace $E_{s}$. It uses some rotational invariance properties founded naturally in the case of exponential. A decomposition of the matrix $\mathrm{S}$ into two matrices $\mathrm{S}_{1}$ and $\mathrm{S}_{2}$ is considered as follows:

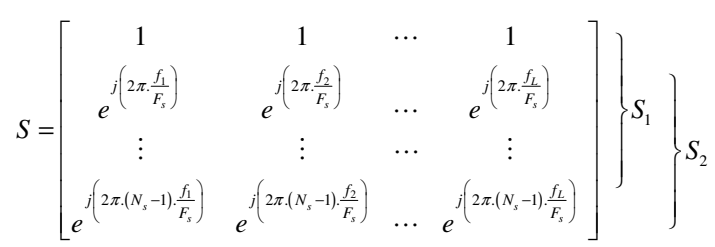

$S_{I}$ represents the first $N_{s}-1$ rows of the matrix S

$S_{2}$ represents the last $N_{s}-1$ rows of the matrix S

The rotational invariance between both subspaces leads to the equation:

$$
S_{1}=\Phi S_{2}
$$

The matrix $\Phi$ contains all informations about $L$ components frequencies. Nevertheless, the estimated matrices $S$ can contain errors. Thereafter, the ESPRIT-TLS (total least-squares) algorithm finds the matrix $\Phi$ by minimization of matrix error given by [20]: 


$$
\Phi=\left[\begin{array}{cccc}
e^{j\left(2 \pi \cdot \frac{f_{1}}{F_{s}}\right)} & 0 & \cdots & 0 \\
& e^{j\left(2 \pi \cdot f_{2}\right)} & \cdots & 0 \\
\vdots & \vdots & \ddots & \vdots \\
0 & 0 & \cdots & e^{j\left(2 \pi \cdot \frac{f_{L}}{F_{s}}\right)}
\end{array}\right]
$$

The determination of this matrix can lead to obtain the frequency estimates defined by this formula:

$$
f_{k}=\frac{\operatorname{Arg}\left(\Phi_{k, k}\right)}{2 \pi} F_{s}, k=1,2, \ldots, L
$$

\subsection{Harmonics Powers Estimation}

Once the searched frequencies components of the signal are estimated by ESPRIT, the values of their amplitudes and then their powers can be estimated. By using the eigendecomposition of the subspace signal [17], [19]:

$$
R_{s}=S \cdot P \cdot S^{H}=\sum_{k=1}^{L}\left(\lambda_{k}+\sigma_{b}^{2}\right) \cdot v_{k} \cdot v_{k}^{H}
$$

It is assumed that the eigenvectors of the signal subspace are normalized as follows:

$$
v_{k}^{H} \cdot v_{k}=1
$$

Thus, for $k=1,2, \ldots, L$ :

$$
R_{i} \cdot v_{k}=\lambda_{k} \cdot v_{k}
$$

Multiplying both sides of this equation by $v_{\mathrm{k}}{ }^{\mathrm{H}}$ :

$$
v_{k}^{H} \cdot R_{i} \cdot v_{k}=\lambda_{k} \cdot v_{k}^{H} \cdot v_{k}
$$

According to (4), (11) and (21):

$$
v_{k}^{H} \cdot R_{i} \cdot v_{k}=v_{k}^{H} \cdot\left[\sum_{k=1}^{L} P_{k} s_{k} s_{k}^{H}+\sigma_{b}^{2} \cdot I\right] \cdot v_{k}=\lambda_{k}
$$

This equation can be simplified as follows:

$$
\sum_{k=1}^{L} P_{k} \cdot\left|s_{k}^{H} \cdot v_{k}\right|^{2}=\lambda_{k}-\sigma_{b}^{2}
$$

Using the following equation:

$$
\left|s_{k}^{H} \cdot v_{k}\right|^{2}=\left|Q_{k}\left(e^{j 2 \pi f_{k}}\right)\right|^{2}
$$

Equation (22) can be written in the following expression:

$$
\sum_{k=1}^{L} P_{k} \cdot\left|Q_{k}\left(e^{j 2 \pi f_{k}}\right)\right|^{2}=\lambda_{k}-\sigma_{b}^{2}
$$

This equation is a set of $L$ linear equations with a number $L$ of unknown harmonics powers. It is very easy to extract the harmonics powers vector $P$ from the equation (25) by simple resolution. 


\section{IMPROVED ESPRIT METHOD}

The discrimination of all small amplitude frequency components around $f_{0}$ by ESPRIT method is difficult. This is mainly due to the significant computation time elapsed by this algorithm to find harmonic sideband components correctly. Furthermore, ESPRIT calculation cost increases when the size of the autocorrelation matrix and the number of data samples increase. It depends on the complexity of $N_{s}{ }^{3}$. This delay forms a major drawback that can cause a catastrophic evolution of a wind turbine fault which may lead to greatest damages. In order to apply a proactive, robust and real time wind turbine condition monitoring, an improved version of ESPRIT algorithm entitled Fast-ESPRIT was used. The ameliorated algorithm is based on both a band-pass IIR filtering and decimation technique in the fault frequency bandwidth $\left[f_{l}, f_{h}\right]$, where $f_{l}$, $f_{h}$ are the low cut-off and high cut-off frequency of the band-pass filter. This process provides a remarkable reduction in computation time and in data memory size. The decimation factor used in this research is computed with respect to the Nyquist criteria as follows [21]:

$$
\Gamma=\left\{\begin{array}{l}
\frac{F_{\text {Nyquist }}}{2 f_{0}}=\frac{F_{s}}{4 f_{0}} \text { if } f_{h}<95 \mathrm{~Hz} \\
\frac{F_{\text {Nyquist }}}{6 f_{0}}=\frac{F_{s}}{12 f_{0}} \text { if } 95 \mathrm{~Hz} \leq f_{h}<500 \mathrm{~Hz}
\end{array}\right.
$$

Figure 1 shows the block diagram scheme of different stages that Fast-ESPRIT algorithm must executes to identify the fault harmonic frequencies and their powers.

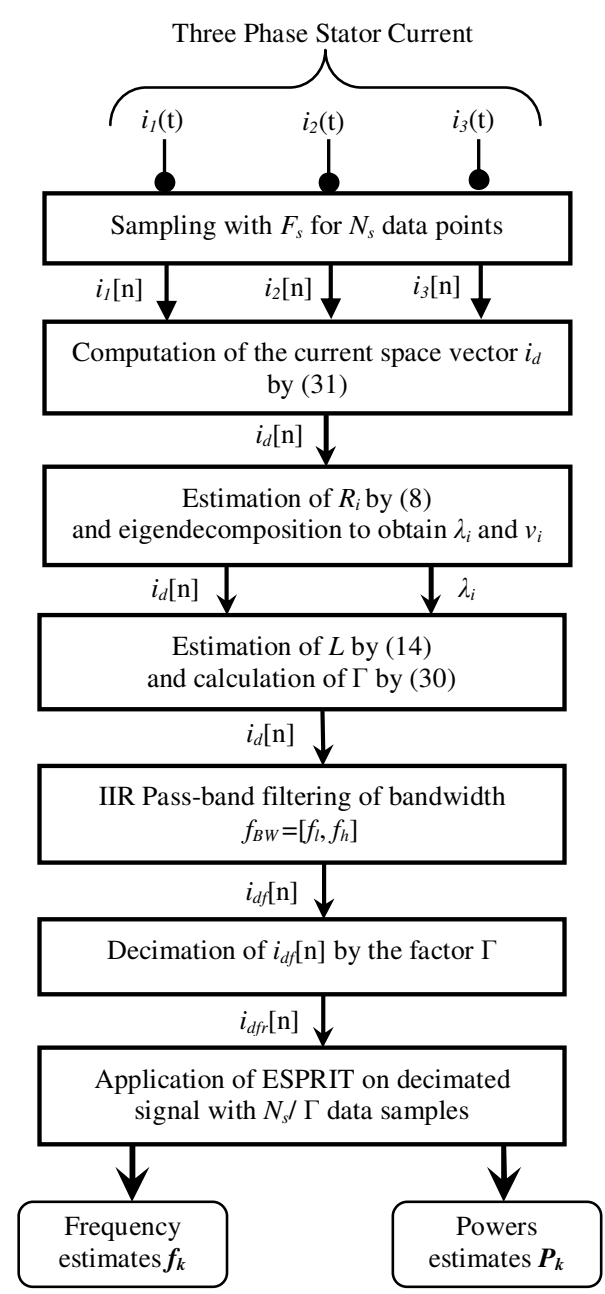

Figure 1. Block diagram scheme of the Fast-ESPRIT algorithm 
Figure 2 shows the variation of $\Gamma$ according to $f_{h}$. The decimation factor decreases with the increase of the maximum harmonics frequency detected in the signal.

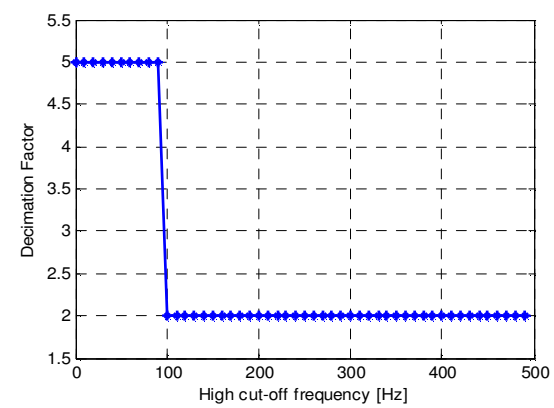

Figure 2. Evolution of decimation factor with fault frequency

In the first time, the acquired sequences $i_{1,2,3}[n]$ of the three phase stator current sampled at the frequency $F_{s}$, are used to calculate the stator current space vector as follows [22]:

$$
i_{d}=\frac{i_{1}+a \cdot i_{2}+a^{2} \cdot i_{3}}{3}, a=e^{j \frac{2 \pi}{3}}
$$

Where $a, a^{2}$ are the spatial operators. This vector allows a fault diagnosis on all phase stator current instead of examining fault signature on each ones. With this method computation time will be minimized. In the second step, an estimation of the autocorrelation matrix $R_{i}$ is realized and therefore the eigenvalues $\lambda_{i}$ are extracted to estimate the number of researched harmonics $L$ in the stator current signal with respect to $M D L$ criterion seen in (14). Then, the signal sequence $i_{d}[n]$ is filtered via a recursive Infinite Impulse Response (IIR) digital band-pass filter based on a least squares fit in the frequency range $\left[f_{l}, f_{h}\right]$ characterizing the fault. This filter has a flat response in the desired bandwidth and its use is justified by the fact that it will be helpful to extract just the informations contained in the signal which are useful in the fault recognition which can occurs at any time. In the third stage, the received sequence of the stator current space vector $i_{d f}[n]$ is decimated by a factor $\Gamma$ shown in (30). In addition, the applied decimation uses low-pass filter to ensure antialiasing. The motivation for this decimation is to reduce the cost processing and memory required for a cheaper implementation. Finally, the ESPRIT algorithm is applied on the decimated signal sequence having $N_{s} / \Gamma$ data samples to identify all frequency components and their powers contained in the signal.

\section{SIMULATION RESULTS AND ANALYSIS}

The developed approach seen in the previous section has been applied and simulated under different scenarios of wind turbine fault types shown in Table 1. To evaluate its performance in real time fault detection, Fast-ESPRIT algorithm has been integrated with a fault diagnosis controller which coordinates with vibration sensors localized in specific wind turbine mechanical components to monitor vibration levels. The controller decides and classifies the existence of a fault depending on vibration measurements collected by the sensors and the harmonic frequencies with their powers estimated by the Fast-ESPRIT method. Figure 2 illustrates the explained technique. Besides, the applied diagnosis algorithm is based on the use of a fault frequency band switching which sweeps any prospective faults that may occur and subsequently classify them by type according to their frequencies. Thus, the diagnosis is made by the intervals of the spectrum reflecting the signature of a possible default [23], [25]. This means that the Fast ESPRIT method will not be applied to the entire signal but only on a part that contains the target information to be extracted for analysis. In case of fault detection, a system alarm is triggered to alert monitoring and maintenance staff for an emergency intervention repair. This procedure provides many benefits because it allows high recognizing and classification of faults with economic and real time implementation [24]. Computer simulations are realized in Matlab for a faulty wind turbine generator using 2 pair poles, $4 \mathrm{~kW} / 50 \mathrm{~Hz}, 230 / 400 \mathrm{~V}$. 


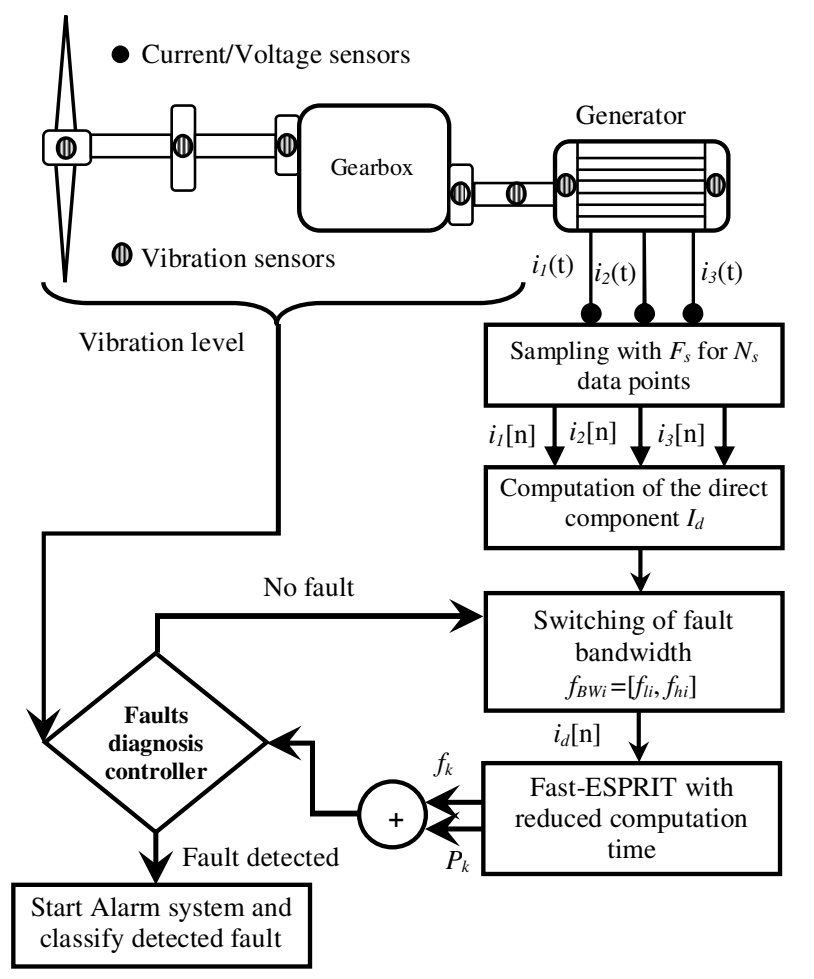

Figure 3. Intelligent wind turbine faults diagnosis by Fast-ESPRIT

The induction generator stator current, is simulated by using the signal model described in (1) for the different failure kinds described in Table 1. The parameters of the simulations are illustrated in Table 2.

Table 2. Parameters used in the simulations

\begin{tabular}{cc}
\hline Parameter & Value \\
\hline $\mathrm{s}$ & 0,033 \\
$\mathrm{P}$ & 2 \\
$f_{0}$ & $50 \mathrm{~Hz}$ \\
$f_{\mathrm{r}}$ & $29,01 \mathrm{~Hz}$ \\
$\mathrm{n}_{\mathrm{b}}$ & 12 \\
$\mathrm{~N}_{\mathrm{s}}$ & 1024 \\
$\mathrm{~F}_{\mathrm{s}}$ & $1000 \mathrm{~Hz}$ \\
Fundamental Stator Current & $10 \mathrm{~A}$ \\
Amplitude & Intel Core2 Duo T6570 \\
Computation Processor & $2,1 \mathrm{GHz}$ \\
\hline
\end{tabular}

To simplify the simulation, a single phase of the generator stator current has been studied. The power of each fault is calculated based on its amplitude as follows:

$$
P_{k}=10 \log _{10}\left(\frac{I_{k}}{2}\right)
$$

Before examining the stator current signal, it must be filtered to obtain in the output a composite signal having a totally negligible noise compared to the fundamental and its harmonics. 


\subsection{Air Gap Eccentricity Detection}

Table 4 shows the simulation results for identifying wind turbine generator air gap eccentricity fault signature in the goal to compare the performance of the original ESPRIT-TLS with the proposed FastESPRIT. The harmonics characterizing this fault are given by Table 3.

Table 3. Air gap eccentricity fault parameters

\begin{tabular}{ccccc}
\hline$f_{\text {ecc }}(\mathrm{Hz})$ & $I_{\text {ecc }}(\mathrm{A})$ & $P_{\text {ecc }}(\mathrm{dB})$ & $\mathrm{N}_{\mathrm{h}}$ & $\mathrm{SNR}(\mathrm{dB})$ \\
\hline 25.825 & 0.4 & -10.97 & 3 & 80 \\
74.175 & 0.3 & -13.46 & 3 & \\
\hline
\end{tabular}

This experiment was done with a high signal to noise ratio to determine the computing time and the required memory size in both algorithms.

Table 4. Computation performance comparison

\begin{tabular}{cccccc}
\hline Method & $\begin{array}{c}\text { Data } \\
\text { samples }\end{array}$ & $\begin{array}{c}\text { Harmonics } \\
f_{k} / P_{k}\end{array}$ & $\begin{array}{c}\text { Signal Memory } \\
\text { size }(\mathrm{KB})\end{array}$ & $\mathrm{M}$ & Time (s) \\
\hline Original & \multirow{2}{*}{1024} & $\begin{array}{c}50.00 \mathrm{~Hz} / 16.99 \mathrm{~dB} \\
25.82 \mathrm{~Hz} /-10.97 \mathrm{~dB}\end{array}$ & 16 & 511 & 4.3471 \\
ESPRIT & & $\begin{array}{l}74.17 \mathrm{~Hz} /-13.47 \mathrm{~dB} \\
49.99 \mathrm{~Hz} / 16.96 \mathrm{~dB}\end{array}$ & & & \\
Fast & \multirow{2}{*}{205} & $\begin{array}{l}25.81 \mathrm{~Hz} /-12.10 \mathrm{~dB} \\
74.17 \mathrm{~Hz} /-14.12 \mathrm{~dB}\end{array}$ & 3.2 & 102 & \multirow{2}{*}{0.03046} \\
ESPRIT & & & & & \\
\hline
\end{tabular}

It is very clear from table 4 that both original and fast ESPRIT algorithms provide satisfactory accuracy, and they correctly identify the $L=3$ harmonics despite with smallest powers case. The little performance difference observed in the Fast- ESPRIT is justified by the attenuations caused by the IIR band pass filter used. Furthermore, the obtained results confirms the important reduction of the computational time with 142.7 times, the memory size required for processing with 5 times and complexity has been changed from $N_{\mathrm{s}}{ }^{3}$ to $\left(N_{\mathrm{s}} / \Gamma\right)^{3}$. In addition, a negligible performance loss is observed in the power and frequency estimation caused by the band pass filter attenuations. Figure 4 illustrates the estimation of the signal subspace dimension by means of the Rissanen criteria based on $M D L$ function cost shown in (12) and (14).

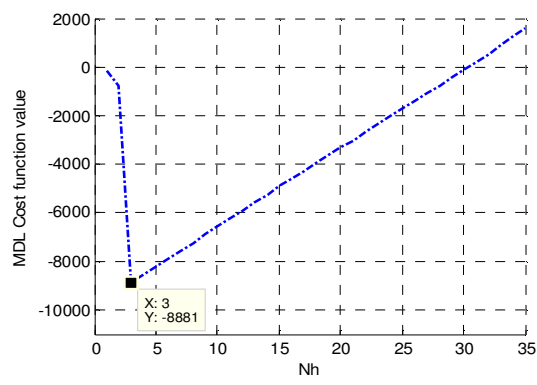

Figure 4. Estimation of signal harmonics number by MDL criterion

Figure 5 shows the frequency response gain of the Yule-Walk IIR band pass filter used in the FastESPRIT algorithm having an order $h=25$. Obviously, the filter has a flat response in the bandwidth target. 


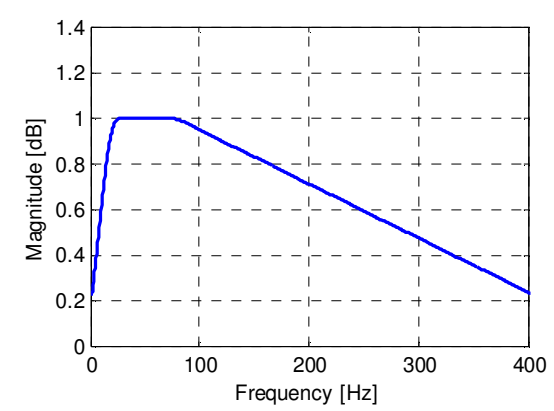

Figure 5. IIR filter Yule-Walk frequency response gain

Whereas, Figure 6 illustrates graphically the power and frequency estimates given by the proposed method. It seems obviously that Fast-ESPRIT has detects all harmonics existents in the eccentricity fault range $[20,80] \mathrm{Hz}$ with high precision.

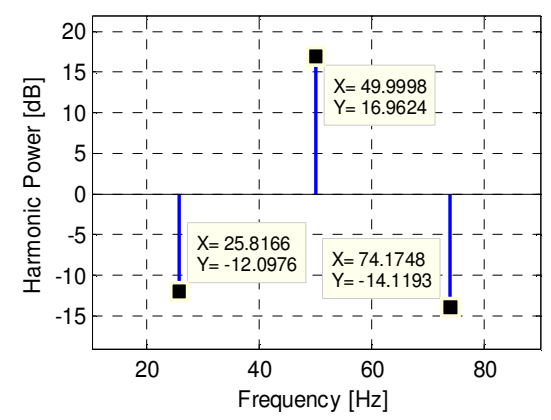

Figure 6. Power and Frequency estimates by Fast-ESPRIT for eccentricity fault

\subsection{Broken Rotor Bars Detection}

The proposed Fast-ESPRIT method has been applied for a wind turbine generator stator current to detect broken rotor bars fault signature in the frequency range $[15,80] \mathrm{Hz}$. The characteristics of this fault harmonics are shown in table 5 . The obtained results are averaged over fifty realizations.

Table 5. Broken rotor bars fault parameters

\begin{tabular}{ccccc}
\hline$f_{\text {brb }}(\mathrm{Hz})$ & $I_{\text {brb }}(\mathrm{A})$ & $P_{\text {brb }}(\mathrm{dB})$ & $\mathrm{N}_{\mathrm{h}}$ & $\mathrm{SNR}(\mathrm{dB})$ \\
\hline 22.525 & 0.3 & $-13.47-$ & & \\
25.825 & 0.45 & $9.95-12.13$ & 5 & 20 \\
70.875 & 0.35 & -10.97 & & \\
74.175 & 0.4 & & \\
\hline
\end{tabular}

From figure 7, the Fast ESPRIT method flows a large calculation time with a large average estimation error rate for discriminating the fault harmonics and their powers contained in a very noisy stator current signal having SNR value less than or equal to $15 \mathrm{~dB}$. This can be interpreted by the delays caused by the algorithm search to find the eigenvalues and eigenvectors of the autocorrelation matrices in both signal and noise spaces. Contrariwise, the calculation time in question decreases gradually for a slightly noisy signal with $\mathrm{SNR}>15 \mathrm{~dB}$. The average estimation error declines also to fall to a minimum value for large values having $\mathrm{SNR}>55 \mathrm{~dB}$. 


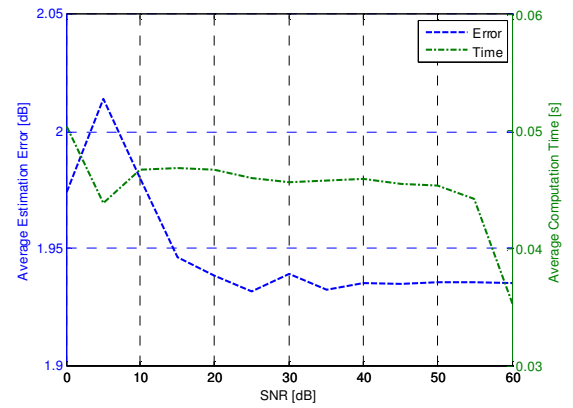

Figure 7. Evolution of fault powers estimation error and computation time with SNR

The method finds difficulty in identifying faults in a very noisy environment. As shown in figure 8, for a stator current signal with a high annoying noise $\mathrm{SNR}<10 \mathrm{~dB}$, detection fault powers presents a considerable error and a remarkable instability level. This error rate decreases when the order of the YuleWalk IIR band pass filter increases. However, the identification performance improves when signal to noise ratio SNR exceeds $10 \mathrm{~dB}$. In this case, the average error rate estimation stabilizes gradually to reach an asymptotic value.

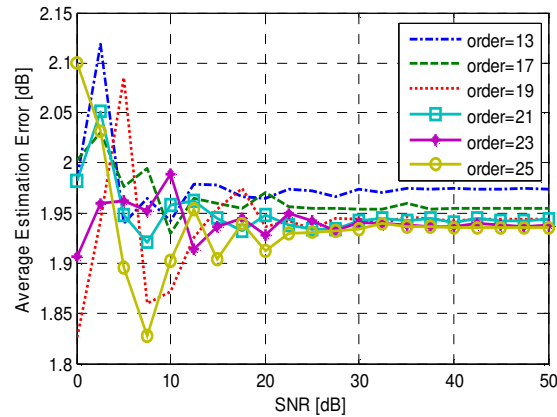

Figure 8. Variation of fault powers estimation error depending on IIR filter order and SNR

Although the standard deviation of the average estimation error is low, thus by increasing the order of the Yule-Walk IIR band pass filter, the accuracy of the method is improved proportionately. By analyzing Figure 9, the fault harmonics discrimination having low amplitudes is so difficult because the power average estimation error reaches a maximum value especially when the SNR decreases. Thus a satisfactory recognition results requires an SNR greater than $15 \mathrm{~dB}$.

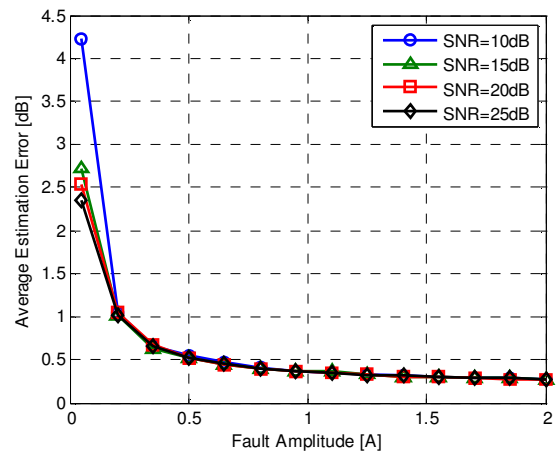

Figure 9. Variation of fault powers estimation error according to fault amplitude and SNR

Against, when the fault magnitude increases the algorithm becomes able to track automatically the harmonics more accurately. 


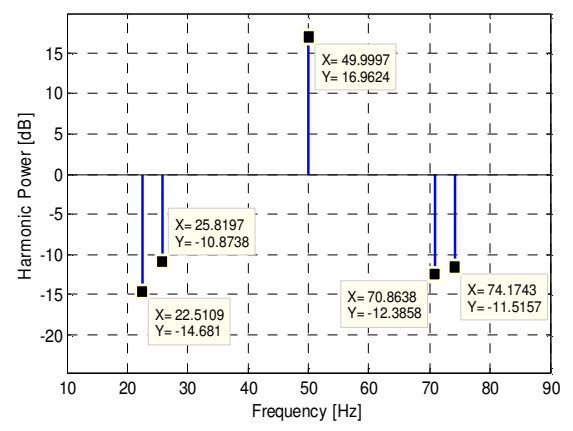

Figure 10. Power and Frequency estimates by Fast-ESPRIT for broken rotor bars fault

Figure 10 provides the achieved detection by the proposed method of broken rotor bars fault harmonics. It is noted that the Fast-ESPRIT algorithm was able to separate spectral components much closer and lower accurately in an optimal computation time which equal to 0.03021 second.

\subsection{Bearing Damage Detection}

In the third test, the proposed algorithm has been used to detect bearing damage fault signature in the frequency range $[40,200] \mathrm{Hz}$. The Table 6 gives the simulated fault parameters.

Table 6. Bearing damage fault parameters

\begin{tabular}{ccccc}
\hline$f_{\text {bng }}(\mathrm{Hz})$ & $I_{\text {bng }}(\mathrm{A})$ & $P_{\text {bng }}(\mathrm{dB})$ & $\mathrm{N}_{\mathrm{h}}$ & $\mathrm{SNR}(\mathrm{dB})$ \\
\hline 89.248 & 0.2 & -16.99 & 3 & 20 \\
155.248 & 0.25 & -15.05 & 3 & \\
\hline
\end{tabular}

Figure 11 shows that the proposed approach provides a satisfactory result with high accuracy with a minimum computation cost which reach 0.07093 second even if the frequency range is wide. It is noted that this computation time is the twice compared to the time required to detect the previous faults.

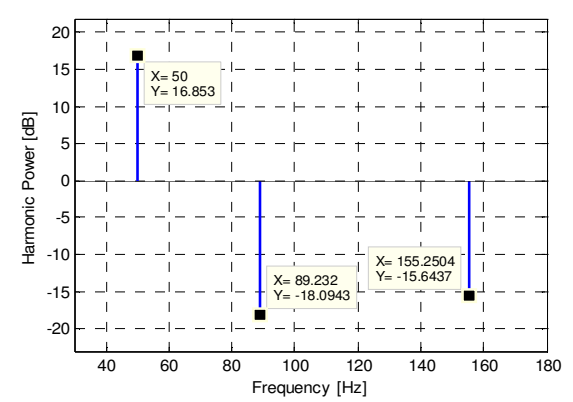

Figure 11. Power and Frequency estimates by Fast-ESPRIT for bearing damage fault

\subsection{Misalignment Detection}

In this simulation, Fast-ESPRIT method has been evaluated to identify an important number of harmonics charactering misalignment fault signature in the frequency bandwidth $[10,210] \mathrm{Hz}$.

Table 7. Misalignment fault parameters

\begin{tabular}{ccccc}
\hline$f_{\text {mis }}(\mathrm{Hz})$ & $I_{\text {mis }}(\mathrm{A})$ & $P_{\text {mis }}(\mathrm{dB})$ & $\mathrm{N}_{\mathrm{h}}$ & $\mathrm{SNR}(\mathrm{dB})$ \\
\hline 21 & 0.22 & -16.16 & & \\
37.03 & 0.33 & -12.64 & & \\
79 & 0.27 & -14.38 & 7 & 20 \\
95.05 & 0.37 & -11.65 & & \\
137.03 & 0.18 & -17.90 & & \\
195.05 & 0.15 & -19.49 & & \\
\hline
\end{tabular}


Referring to the figure 12 , the applied method offers good estimation ability with a very good computation cost equal to 0.07178 second.

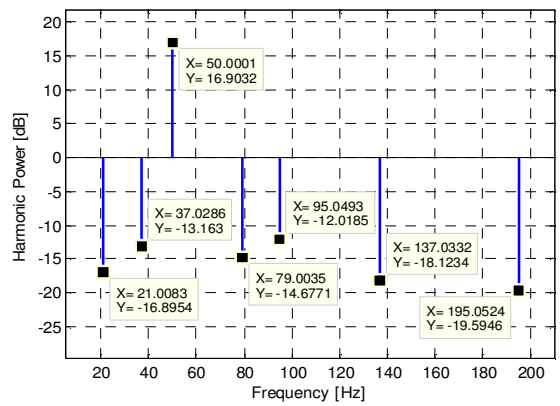

Figure 12. Power and Frequency estimates by Fast-ESPRIT for misalignment fault

As illustrated in the figure 13 , when the frequency bandwidth $\left[f_{l}, f_{h}\right]$ characterizing a fault contains an upper bound $f_{h}$ which is increased and approaching or exceeding the value $F_{N y q u i s t} / 2=F_{\mathrm{s}} / 4$, the decimation factor $\Gamma$ decreases and thereafter the signal data samples increases. This causes the increase of the signal autocorrelation matrix dimension. Consequently this leads to a large calculation time. On the other side, if $f_{h}<F_{\text {Nyquist }} / 2$ the computation time required by the Fast ESPRIT algorithm becomes minimal and it is almost without a big change despite the increase of the fault harmonics number contained in the stator current signal. This increase influences slightly on the calculation time which can be calculated as follows:

$$
T_{c}=\Delta t \frac{N_{s}}{\Gamma}
$$

Where $\Delta \mathrm{t}$ is the time required to process one data sample.

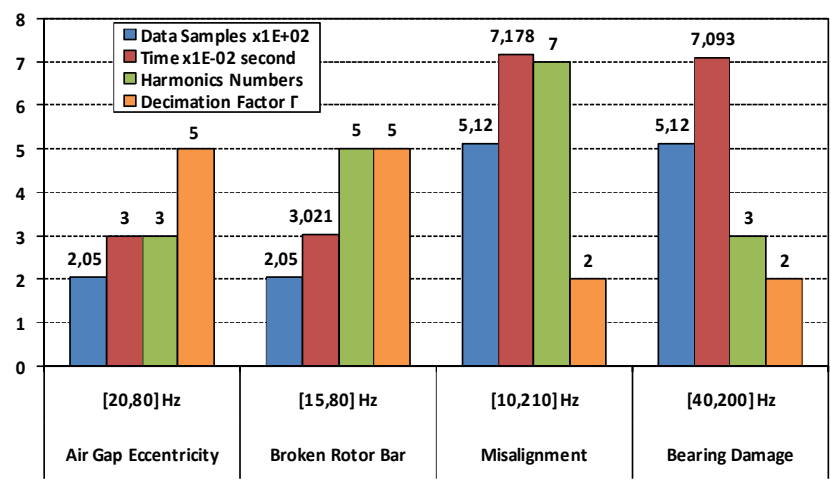

Figure 13. Computation time depending on Fault Frequency range and decimation factor

Another remark that can be added to this interpretation is that the increase of $F_{s}$ leads to increase the size of the signal data samples. This causes the augmentation of the autocorrelation matrix dimension. As result, the Fast-ESPRIT method takes a long time to discriminate all the frequencies contained in the processed signal with an acceptable accuracy. So in order to adapt accurately the detection algorithm to a real-time application, there is a trade-off between the choice of an optimal sampling frequency on one side and the computation time in the other side.

\section{CONCLUSION}

ESPRIT method has better performances than others super resolution algorithms for identifying frequencies from a short data signal acquisition drowned in a noise. However, the main drawback of this technique is the high computational time especially when the size of the signal autocorrelation matrix 
increases. A new version of the ESPRIT algorithm is proposed in this paper entitled Fast-ESPRIT. An improvement is realized with an adequate IIR band pass filtering and an optimal decimation technique. This enhancement leads to low complexity, to satisfactory accuracy and to memory storage reduction algorithm. The proposed technique was applied under different wind turbine faults to evaluate its resolution ability. Analysis of the simulation results shows that estimator achieves remarkable performance estimation in extracting frequencies and amplitudes in a specified bandwidth than the original ESPRIT. Moreover, due to its advantages, Fast-ESPRIT method can be implementable for real time fault diagnosis. The future work will be focused to ameliorate Fast-ESPRIT detection efficiency in the case of low amplitudes harmonics.

\section{REFERENCES}

[1] Hamid A. Toliyat et al. Electric Machines Modeling, Condition Monitoring, and Fault Diagnosis. CRC Press Taylor \& Francis Group NW. 2013.

[2] ML Sin, WL Soong, N Ertugrul. On-Line Condition Monitoring and Fault Diagnosis - A Survey. Australian Universities Power Engineering Conference, New Zealand. 2003.

[3] KK Pandey et al. Review on Fault Diagnosis in Three-Phase Induction Motor. MEDHA, Proceedings published by International Journal of Computer Applications (IJCA). 2012.

[4] E Al Ahmar et al. Advanced Signal Processing Techniques for Fault Detection and Diagnosis in a Wind Turbine Induction Generator Drive Train: A Comparative Study. IEEE Energy Conversion Congress and Exposition ECCE 2010, Atlanta United States. 2010.

[5] John L. Semmlow. Biosignal and Biomedical Matlab-Based Applications. Marcel Dekker, Inc New York. 2004.

[6] Neelam Mehala et al. Condition monitoring methods, failure identification and analysis for Induction machines. International Journal of Circuits, Systems and Signal Processing. 2009; 1(3): 10-17.

[7] Gérard Blanchet and Maurice Charbit. Digital Signal and Image Processing using Matlab. ISTE USA 2006.

[8] Yassine Amirat et al. Wind Turbine Bearing Failure Detection Using Generator Stator Current Homopolar Component Ensemble Empirical Mode Decomposition. IECON 2012 - 38th Annual Conference on IEEE Industrial Electronics Society

[9] Elie Al-Ahmar et al. Wind Energy Conversion Systems Fault Diagnosis Using Wavelet Analysis. International Review of Electrical Engineering. http://hal.univ-brest.fr/docs/00/52/65/07/PDF/IREE_2008_AL-AHMAR.pdf 2008; 3: 646-652.

[10] El Houssin El Bouchikhi, Vincent Choqueuse, MEH Benbouzid. Non-stationary spectral estimation for wind turbine induction generator faults detection. Industrial Electronics Society, IECON 2013-39th Annual Conference of the IEEE. 2013: 7376-738

[11] Ioannis Tsoumas et al. A Comparative Study of Induction Motor Current Signature Analysis Techniques for Mechanical Faults Detection, SDEMPED - International Symposium on Diagnostics for Electric Machines. Power Electronics and Drives Vienna, Austria. 2005.

[12] Yong-Hwa Kim. High-Resolution Parameter Estimation Method to Identify Broken Rotor Bar Faults in Induction Motors. IEEE Transactions on Industrial Electronics. 2013; 60(9): 4103-4117.

[13] Shahin Hedayati Kia et al. A High-Resolution Frequency Estimation Method for Three-Phase Induction Machine Fault Detection. IEEE Transactions on Industrial Electronics. 54(4).2007

[14] Saad Chakkor et al. Performance Analysis of Faults Detection in Wind Turbine Generator Based on HighResolution Frequency Estimation Methods. International Journal of Advanced Computer Science and Applications, SAI Publisher. 2014; 5(4): 139-148.

[15] Jian Zhang et al. Rank Reduced ESPRIT Techniques in the Estimation of Principle Signal Components. Proceedings 5th Australian Communications Theory Workshop, Australian National University, 2004.

[16] Shawn Sheng and Jon Keller et al. Gearbox Reliability Collaborative Update. NREL U.S. Department of Energy, http://www.nrel.gov/docs/fy14osti/60141.pdf

[17] J Proakis, D Manolakis. Digital Signal Processing: Principles, Algorithms, and Applications. New York: Macmillan Publishing Company. 1992.

[18] André Quinquis. Digital Signal Processing using MATLAB. ISTE Ltd, London UK, 2008.

[19] Monson H Hayes. Statistical Digital signal processing and modeling. John Wiley \& Sons, New York, 1996.

[20] R Roy, T Kailath. ESPRIT-estimation of signal parameters via rotational invariance techniques. IEEE Trans. Acoust., Speech, Signal Processing. 1989; 37(7): 984-995.

[21] Fredric J Harris. Multirate Signal Processing for Communication Systems. Prentice Hall. 2004.

[22] Joao Paulo CL da Costa et al. Comparison of Model Order Selection Techniques for High Resolution Parameter Estimation Algorithms. 54th Internationales Wissenschaftliches Kolloquium, Technische Universität Ilmenau, Germany, 2009.

[23] Janos J Gertler. Fault Detection and Diagnosis in Engineering Systems Basic concepts with simple examples. Marcel Dekker Inc., New York. 1998.

[24] Saad Chakkor et al. Wind Turbine Fault Detection System in Real Time Remote Monitoring. International Journal of Electrical and Computer Engineering (IJECE), IAES Publisher. 2014; 4(6).

[25] H Vincent Poor. An Introduction to Signal Detection and Estimation. Second Edition, Springer-Verlag texts in electrical engineering, Virginia USA. 1994. 


\section{BIOGRAPHIES OF AUTHORS}

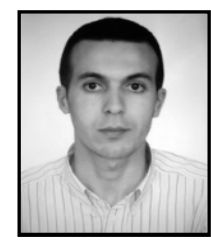

Saad Chakkor was born in Tangier Morocco. He's a member in the Physics department, Communication and detection Systems laboratory, Faculty of sciences, University of Abdelmalek Essaâdi, Tetouan Morocco, and his research area is: wireless intelligent sensors and theirs applications, frequency estimation algorithms for faults detection and diagnosis system in electromecanical machines. He obtained the Master's degree in Electrical and Computer Engineering from the Faculty of Sciences and Techniques of Tangier, Morocco in 2002. He graduated enabling teaching computer science for secondary qualifying school in 2003. In 2006, he graduated from DESA in Automatics and information processing at the same faculty. $\mathrm{He}$ works as teacher of computer science in the high school.

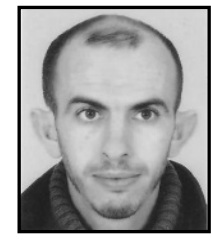

Mostafa Baghouri was born in Tangier Morocco. He's a member in the Physics department, Communication and detection Systems laboratory, Faculty of sciences, University of Abdelmalek Essaâdi, Tetouan Morocco, his research area is: routing and real time protocols for energy optimization in wireless sensors networks. He obtained a Master's degree in Electrical and Computer Engineering from the Faculty of Science and Technology of Tangier in Morocco in 2002. He graduated enabling teaching computer science for secondary qualifying school in 2004. In 2006, he graduated from DESA in Automatics and information processing at the same faculty. He work teacher of computer science in the high school.

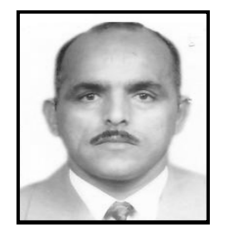

Abderrahmane Hajraoui is a professor of the Higher Education at University of Abdelmalek Essaâdi. He's a director thesis in the Physics department, Communication and detection Systems laboratory, Faculty of sciences, University of Abdelmalek Essaâdi, Tetouan, Morocco. His research areas are: Signal and image processing, automation systems, simulation systems, antennas and radiation, microwave devices and intelligent wireless sensors networks. 